\title{
Dominik Freunberger (Salzburg) Wie Wörter Wellen werden Die Untersuchung von Sprachverarbeitung mittels EEG
}

\begin{abstract}
In diesem Beitrag werden nach einer kurzen methodischen Vorstellung der Elektroenzephalographie und der Ereignis-korrelierten Potenziale einige Eckpunkte, die bei der Gestaltung eines linguistischen EEG-Experimentes Beachtung finden sollten, ausgeführt. Der Beitrag schließt mit Überlegungen, die bei der Untersuchung grammatischer Variation besonders berücksichtigt werden sollten.
\end{abstract}

\section{Warum EEG in der Linguistik?}

Parapsychologie ${ }^{1}$ war der Auslöser, der Hans Berger auf jenen Weg schickte, der am 6. Juli 1924 einen Höhepunkt fand, als er als Erster erfolgreich die elektrische Hirnaktivität des Menschen abgeleitet und auch bereits in Ansätzen funktional interpretiert hat (Berger 1929). Schon damals erahnte man das große Potenzial dieser bildgebenden Methode zur Erforschung neurokognitiver Prozesse.

Die von Berger geprägte Elektroenzephalographie (EEG) ist noch heute die bevorzugte Methode vieler Neurowissenschaftler sämtlicher Forschungsrichtungen. Verantwortlich dafür sind neben den relativ geringen Anschaffungs-, Instandhaltungs- und Betriebskosten und der Anwendbarkeit der Methode auf beinahe alle Teile der Bevölkerung, vermutlich zweierlei Gründe: Zum einen erfasst EEG direkt, unmittelbar und nicht-invasiv elektrochemische Veränderungen von Nervenzellpopulationen und grenzt sich damit von bildgebenden Verfahren ab, die neuronale Aktivität indirekt über physiologische Veränderungen, wie etwa zerebrale Durchblutungsänderungen, messen. Zum anderen verfügt EEG über jene zeitliche Auflösung im Millisekundenbereich, die zur Untersuchung rapide ablaufender neuronaler Prozesse unabdingbar ist.

1 Berger erhielt, als er als Offizier in der preußischen Armee diente, einen Brief seiner Schwester, in welchem sie von einem Traum erzählte. In diesem fiel ihr Bruder Hans vom Pferd und verletzte sich. Ohne dass seine Schwester davon wissen konnte, hatte Berger tatsächlich einen Unfall. Als einzige Erklärung hierfür kam Gedankenübertragung - also Telepathie - in Frage, was wiederum Auslöser war, die elektrische Aktivität des Gehirns zu untersuchen (Wiedemann 1994). 
Dies kommt vor allem bei der Untersuchung sprachlicher Verarbeitungsvorgänge zur Geltung: Zwischen Wortkategorien kann bereits nach etwa 100 Millisekunden (ms) unterschieden werden, die Bedeutung eines Wortes wird schon nach etwa $300 \mathrm{~ms}$ erkannt und syntaktische Integration ist nach etwa $600 \mathrm{~ms}$ abgeschlossen (Friederici 2002). Die Kehrseite der hohen zeitlichen ist die geringe räumliche Auflösung: Während etwa die funktionale Magnetresonanztomographie Veränderungen innerhalb weniger Kubikmillimeter detektieren kann, können die neuronalen Strukturen, die für das gemessene EEG-Signal verantwortlich sind, lediglich auf etwa einen halben Zentimeter eingeschränkt werden - und dies nur auf der Kortexoberfläche (Rösler/Röder/Streb 2003).

Des Weiteren ist EEG als so genannte Online-Methode in der Lage, neuronale Vorgänge quasi in Echtzeit abzubilden. Im Gegensatz dazu können Offline-Methoden, wie etwa Akzeptabilitätsbeurteilungen, lediglich eine Gesamtsatzperspektive erfassen. Das folgende Beispiel soll diesen Unterschied verdeutlichen.

(1) Ina legt die Semmel, die was der Michael mit dem Butter bestrichen hat, auf das Teller.

Abgesehen von Sprechern einer bestimmten Varietät des Deutschen würde der Großteil der Befragten in einem Beurteilungsexperiment den Satz unter (1) vermutlich als ,nicht akzeptabel` einstufen. Es bleibt jedoch schleierhaft, welcher Teil des Satzes die Versuchsteilnehmer/innen veranlasst hätte, den Satz als inakzeptabel zu bewerten. Mit einer Online-Methode hingegen hätte man dem Gehirn quasi beim Verarbeiten des Satzes zusehen und somit messen können, an welcher Satzposition es zu erhöhten Verarbeitungsschwierigkeiten kommt. Man hätte also mögliche Ursachen für die Akzeptabilitätsbeurteilung identifizieren können.

Es ist also nicht verwunderlich, dass sich die von Berger maßgeblich geprägte Methode vor allem in der experimentellen Psycholinguistik nach wie vor solch großer Beliebtheit erfreut. Im folgenden Abschnitt werden erst die biologischen Grundlagen der Elektroenzephalographie umrissen, bevor eine methodische Anwendung des EEG - die so genannten Ereignis-korrelierten Potenziale - beschrieben wird.

\section{Vom Neuron zum EKP}

Welche neuronale Aktivität wird von den Elektroden an der Kopfhaut gemessen? In einem Satz könnte man sagen, EEG misst die aufsummierte, postsynaptische Aktivität parallel ausgerichteter Pyramidenzellen, die perpendikulär zur Skalp- 
oberfläche liegen. Das heißt, EEG detektiert die Aktivität von Zellverbänden und nicht von einzelnen Neuronen. Die dabei aktiven Zellen müssen zudem gleichzeitig aktiv sein, parallel zueinander und im $90^{\circ}$-Winkel zur Schädeloberfläche liegen eine Eigenschaft, die vor allem von Pyramidenzellen erfüllt wird. Kommt es innerhalb einer Nervenzelle zu einem postsynaptischen Potenzial, so entsteht für kurze Zeit ein magnetischer Dipol und es fließt Strom zwischen den beiden Polen. Das dadurch entstehende Magnetfeld wird auf die Kopfhaut übertragen, von wo diese Oszillationen mit Elektroden erfasst werden können (Bornkessel-Schlesewsky/ Schlesewsky 2009). Da das Signal sehr schwach ist, wird es verstärkt (1000100.000-fach) und das kontinuierliche elektrische Potenzial wird zur Aufzeichnung in diskrete (digitale) Werte umgewandelt (Luck 2012).

Dabei gibt es jedoch zwei nennenswerte Einschränkungen: Erstens besteht die Möglichkeit, dass ein Signal zwar vorhanden, jedoch nicht an der Schädeloberfläche messbar ist. Dies kann der Fall sein, wenn sich mehrere Potenziale durch Interferenz auslöschen oder wenn etwa die aktiven Neuronenverbände nicht perpendikulär, sondern parallel zum Skalp liegen. Die Aktivität von tiefer liegenden neuronalen Strukturen, wie etwa der Basalganglien, kann typischerweise ebenfalls nicht erfasst werden. Zweitens unterliegt EEG dem so genannten Inversproblem. Dieses besagt, dass es für jede elektrische Verteilung, die an der Schädeloberfläche gemessen wird, (theoretisch) unendlich viele zugrundeliegende neuronale Aktivitätsmuster geben kann, was natürlich zu einer deutlich eingeschränkten räumlichen Interpretierbarkeit führt (Otten/Rugg 2005).

Eine Anwendung des EEG sind die so genannten Ereignis-korrelierten Potenziale (EKPs; von eng. event-related potentials). EKPs sind elektrische Potenziale, die vom Gehirn relativ zu einem internen oder externen Ereignis generiert wurden. Um aus dem Roh-EEG EKPs zu gewinnen, werden nach mehreren Vorverarbeitungsschritten (z.B. Re-Referenzierung, Filterung, Artefaktkorrektur) aus dem kontinuierlichen EEG-Signal gleich große Segmente um relevante Ereignisse (z.B. bestimmte Wörter, die präsentiert wurden) herausgeschnitten. Man erhält dann beispielsweise Segmente, die 500 ms vor dem relevanten Ereignis beginnen und eine Sekunde nach dem Ereignis enden. Natürlich ist dafür erforderlich, dass die Information über die der Versuchsperson dargebotenen Ereignisse im EEGSignal kodiert ist.

Diese so gewonnenen Segmente werden dann pro Bedingung und Versuchsperson gemittelt. Diese Signalmittelung ist ein wesentlicher Schritt, denn bei ihr werden jene Anteile im Signal, die nicht zeitlich an das Ereignis gebunden sind (also quasi zufällig auftreten), gegen Null gemittelt. Dagegen werden Anteile, die relativ zum Ereignis zeitlich stabil auftreten, verstärkt (Luck 2012).

Das finale Ereignis-korrelierte Potenzial bildet dann die Stromstärke über die Zeit relativ zu einem Ereignis ab. Dieses Signal ist zusammengesetzt aus unter- 
schiedlichen Komponenten, wobei es wichtig ist, auf den Begriff der EKP-Komponente näher einzugehen. Darunter versteht man einen Spannungsverlauf, der durch eine bestimmte neuronale Aktivität in einem bestimmten Areal hervorgerufen wird. Da sich viele dieser Prozesse überlagern, überlagern sich ebenso die von ihnen hervorgerufenen Komponenten. Ein EKP-Verlauf ist daher die Summe vieler, sich überlagernder Komponenten (Luck 2012). Gängige Nomenklatur dieser Komponenten rekurriert auf deren Polarität kombiniert mit deren Amplitudenmaximum, also etwa N100 für eine Negativierung um 100 ms oder P300 für eine Positivierung um $300 \mathrm{~ms}$.

Manche dieser EKP-Komponenten wurden als besonders wichtig für die Untersuchung sprachlicher Phänomene identifiziert. 1980 fanden Marta Kutas und Steven Hillyard eher unerwartet eine EKP-Komponente, die bis heute in engem Zusammenhang mit semantischer Verarbeitung gesehen wird und durch ihr systematisches Auftreten zu den am meist untersuchten überhaupt zählt: die N400. Diese Negativierung mit größter Amplitude um 400 ms nach Präsentation eines Wortes spiegelt vermutlich den Aufwand des Gehirns wider, Information aus dem semantischen Langzeitgedächtnis abzurufen. Ihre Amplitude ist größer, wenn der Abruf schwerer fällt, wie es etwa der Fall ist, wenn Wörter weniger frequent sind, im Kontext unerwartet auftreten oder mit diesem nicht passförmig sind. Begünstigt ein Kontext die Vorhersage eines Wortes, so wird diese Wortinformation (vor-)aktiviert und der eigentliche Abruf der Information nach Sehen oder Hören des Wortes fällt leichter. Seit der bahnbrechenden Entdeckung von Kutas und Hillyard gibt es unzählige Experimente in sämtlichen Modalitäten, die Einflussfaktoren auf die Amplitude der N400 identifizieren konnten (vgl. Kutas/ Federmeier 2011).

Die P600 (also eine Positivierung um $600 \mathrm{~ms}$ ) wird typischerweise durch syntaktische Manipulationen wie grammatische Verletzungen der garden pathKonstruktionen hervorgerufen. Es wird gemutmaßt, sie spiegle Reparatur-, Integrations- oder Reanalyseprozesse wider. Zudem werden auch frühere Negativierungen, wie die (frühe) links-anteriore Negativierung durch Phrasenstruktur- oder Kongruenzverletzungen hervorgerufen. Einen guten Überblick über sprachlich relevante EKPs gibt Kaan (2007).

\section{3 Überlegungen zur Experimentgestaltung}

Plant man ein EKP-Experiment, um eine linguistische Fragestellung zu beantworten, gibt es einige Punkte, die dabei beachtet werden sollten. Auf diese soll im Folgenden jeweils kurz eingegangen werden. 
Fragestellung. An erster Stelle bei der Konzeption eines Experimentes steht die Fragestellung, die ausschlaggebend für die weitere Planung des Experimentes ist. Möchte ich die Verarbeitung einer Konstruktion relativ zu einer anderen oder eine Konstruktion in verschiedenen Kontexten untersuchen? Möchte ich zwei unterschiedliche Populationen miteinander vergleichen? Möchte ich die gleiche Population in unterschiedlichen Kontexten untersuchen? Möchte ich graduelle Unterschiede untersuchen?

Versuchspersonen. Welche Population möchte ich untersuchen? Welche Einflussfaktoren müssen erhoben oder kontrolliert werden? So können unter anderem Alter, Geschlecht, Erst-, Zweitsprache, Erwerbsalter der jeweiligen Sprache, regionale/dialektale Herkunft, Bildungsniveau, Sprachgebrauch, Sprachgebrauch der Eltern oder nahestehender Personen, oder aber kognitive Faktoren, wie IQ, Gedächtnisleistung oder Lesefähigkeit, einen Einfluss auf die Verarbeitung und damit auf die gemessenen Potenziale haben. Außerdem gilt es zu beachten, dass ebenso neurologische oder psychische Erkrankungen, Medikation oder Händigkeit Einflussfaktoren sein können (Schmid 2016).

Stimulusmaterial. Eine wichtige Frage, die bereits vor der konkreten Umsetzung eines Experiments gestellt werden sollte, ist ob man ausreichend Stimulusmaterial generieren kann. Vor allem in der Sprachwissenschaft gibt es oftmals interessante und erforschenswerte Konstruktionen, die jedoch zu spezifisch sind, um genügend Sätze als Stimulusmaterial erstellen zu können (je nach Manipulationstyp idealerweise etwa 10-30 pro Bedingung; Luck 2012). Darüber hinaus gilt zu bedenken, dass EKPs nur relativ interpretierbar sind, das heißt, es bedarf immer einer oder mehrerer Vergleichsbedingungen. In der Regel unterscheidet sich diese lediglich hinsichtlich einer Manipulation, auf welche potenziell gemessene Unterschiede zurückzuführen sind.

Entscheidend für gutes Stimulusmaterial ist die Ermittlung oder die Kontrolle von Stimuluseigenschaften, die die beobachteten Muster beeinflussen können. Auf Wortebene sind dies etwa Wortart, Frequenz, Länge, n-Gramm-Häufigkeit oder Wortform. Auf Satzebene können unter anderem Plausibilität, Natürlichkeit, Akzeptabilität, semantische Relatiertheit oder auch Prädizierbarkeit die Verarbeitung prägen. Daher ist zu beachten, dass für ein gut kontrolliertes Set an Stimulussätzen oftmals mehrere Vortests unumgänglich sind, um oben genannte Stimuluseigenschaften zu testen und zu quantifizieren.

Modalität. Wie werde ich mein Stimulusmaterial präsentieren? Vor allem in der experimentellen Psycho- und Neurolinguistik ist die so genannte rapid-serial visual presentation (RSVP) eine sehr beliebte Präsentationsform. Dabei werden die Wörter eines Satzes einzeln in der Bildschirmmitte präsentiert, was den Vor- 
teil hat, dass man kontrollieren kann, wann die Versuchsperson welchen Stimulus sieht. Neben der zusätzlichen Kontrolle über das Tempo der Präsentation (typischerweise ca. 500 ms), ist RSVP gesprochener Sprache sehr ähnlich, da der Rezipient nicht zurückspringen kann, wie es etwa bei natürlichem Lesen der Fall ist. Möchte man Sprachverarbeitung bei natürlichem Lesen untersuchen, müssen Sätze als Ganzes dargeboten werden. Dabei ist es notwendig parallel zum EEG auch die Blickbewegungen der Versuchsperson zu registrieren, damit später die relevanten Zeitpunkte (z.B. wann ein kritisches Wort fixiert wurde) bestimmt werden können. Entscheidet man sich für auditive Präsentation, sollten Faktoren, wie Aussprache, Geschwindigkeit oder Koartikulation bedacht werden. Die Bestimmung und Kodierung der relevanten Bereiche (z.B. Beginn oder Ende eines Wortes) in auditivem Material können zudem viel Zeit und Aufwand in Anspruch nehmen (Meulman et al. 2016).

Aufgabenstellung. Der Online-Task, das heißt, die Aufgabe, die die Versuchspersonen während des EEG-Experimentes zu bewerkstelligen haben, kann nicht nur das Ergebnis maßgeblich beeinflussen (Roehm et al. 2007), sondern zudem auch wertvolle Hinweise über die Verarbeitung liefern. So können etwa die EEG-Daten relativ zu den Antworten, die während des Experiments gegeben wurden, ausgewertet werden (vgl. Roehm/Sorace/Bornkessel-Schlesewsky 2013). Grundsätzlich wird zwischen zwei Arten von Online-Aufgaben unterschieden: Zum einen kann metasprachliches Wissen (Akzeptabilität, Plausibilität, Grammatikalität, Wahrheitswert, Natürlichkeit etc.) abgefragt werden. Hier gilt $\mathrm{zu}$ beachten, dass viele dieser Konzepte für naive Versuchsteilnehmer/innen unbekannt sind und daher indirekt erfragt werden müssen (z.B. kann Plausibilität mittels der Frage „Wie sehr stimmen Sie mit der Aussage des Satzes überein?“ erhoben werden).

Zum anderen gibt es Aufgaben, die das aufmerksame Lesen der Sätze sicherstellen sollen, zum Beispiel mittels eines so genannten Probe-Tasks, bei dem die Versuchspersonen bestimmen sollen, ob ein Wort im vorhergehenden Satz enthalten war oder nicht, oder etwa mittels inhaltlicher Verständnisfragen. Gibt es zum Beispiel einen Probe-Task, so werden für die EEG-Auswertung oftmals nur jene Durchgänge benutzt, in denen die Probanden und Probandinnen korrekt geantwortet haben. Es soll außerdem überlegt werden, ob die Antwortmöglichkeiten binär sein oder auf einer Skala liegen sollen und ob die Probanden schnellst- oder bestmöglich antworten sollen.

Nach diesen kleinen Denkanstößen, die die Konzeption, Implementierung und Durchführung eines Experimentes erleichtern können, werden nun im letzten Abschnitt einige dieser Punkte aufgegriffen und im Kontext der Untersuchung grammatischer Variation neu beleuchtet. 


\section{Die Untersuchung grammatischer Variation}

Neben oben genannten Überlegungen, die für jedes Experiment angestrengt werden sollten, gibt es einige Besonderheiten, die bei der Untersuchung grammatischer Variation zusätzlich bedacht werden sollten. Diese betreffen überwiegend Variation auf Stimulus- sowie Versuchspersonenebene.

Material. Wie wird entschieden, was Test- und was Kontrollbedingung ist? Ist die Standardvarietät tatsächlich eine geeignete Vergleichsbedingung? Gibt es Konstruktionen, die hinsichtlich ihrer funktionalen Interpretation variieren? Wie erziele ich die gewünschte funktionale Lesart? Bei auditiven Experimenten ist zudem die Rolle des Sprechers zu beachten: Ist dieser geeignet für eine bestimmte (dialektale) Varietät? Wie können qualitative Eigenschaften einer Aussprachevarietät in messbare Größen umgeformt werden? Wie können gruppenabhängige Stimuluseigenschaften, wie etwa regions-, alters-, oder gruppenspezifische Frequenz von Wörtern sowie Häufigkeiten von Aussprachemustern, bestimmt werden? Kann die Varietät schriftsprachlich untersucht werden oder bedarf es auditiver Präsentation?

Versuchspersonen. Finde ich genügend Versuchspersonen mit gewünschten Eigenschaften (z.B. Sprecher einer bestimmten Varietät)? Wie bestimmt man, ob eine Versuchsperson geeignet, etwa mit einer Varietät vertraut ist? Kann und soll man weitere Einflussvariablen kontrollieren (z.B. passiver versus aktiver Gebrauch, Herkunft, Umgebungsvariablen etc.)? Soll das sprachliche Wissen direkt oder indirekt abgefragt werden? Kann ich mich auf die Angaben der Versuchspersonen verlassen?

Ein weiterer, möglicherweise kritischer Faktor ist die experimentelle Situation: Ist man interessiert an der Untersuchung situationsabhängiger Varietäten, so muss man bedenken, dass ein experimentelles Setting meist sehr formal erscheint. Versucht man in dieser Versuchsumgebung ein informelles Setting zu schaffen, so ist darauf zu achten, eine gewisse Natürlichkeit der Präsentation zu bewahren.

Trotz dieser zusätzlichen Faktoren, die berücksichtigt werden sollten, erscheint die Elektroenzephalographie als eine ausgezeichnete Methode, um die neurokognitiven Mechanismen, die der Verarbeitung grammatischer Variation zugrunde liegen, zu erforschen. 


\section{Literatur}

Berger, Hans (1929): Über das Elektrenkephalogramm des Menschen. In: European Archives of Psychiatry and Clinical Neuroscience 87, S. 527-570.

Bornkessel-Schlesewsky, Ina/Schlesewsky, Mattias (2009): Processing syntax and morphology: A neurocognitive perspective. (= Oxford Surveys in Syntax and Morphology 6). Oxford.

Friederici, Angela D. (2002): Towards a neural basis of auditory sentence processing. In: Trends in Cognitive Sciences 6, S. 78-84.

Kaan, Edith (2007): Event-related potentials and language processing: A brief overview. In: Language and Linguistics Compass 1, S. 571-591.

Kutas, Marta/Federmeier, Kara D. (2011): Thirty years and counting: Finding meaning in the N400 component of the event related brain potential (ERP). In: Annual Review of Psychology 62, S. 621-647.

Kutas, Marta/Hillyard, Steven A. (1980): Reading senseless sentences: Brain potentials reflect semantic incongruity. In: Science 207, S. 203-205.

Luck, Steven J. (2012): Event-related potentials. In: Cooper, Harris M. (Hg.): APA handbook of research methods in psychology. Bd. 1: Foundations, planning, measures, and psychometrics. Washington, S. 523-546.

Meulman, Nienke et al. (2016): EEG and event-related brain potentials. In: Schmid, Monika et al., S. 81-107.

Otten, Leun J./Rugg, Michael D. (2005): Interpreting event-related brain potentials. In: Handy, Todd C. (Hg.): Event-related potentials: A methods handbook. Cambridge, MA, S. 3-16.

Roehm, Dietmar et al. (2007): To predict or not to predict: Influences of task and strategy on the processing of semantic relations. In: Journal of Cognitive Neuroscience 19, S. 1259-1274.

Roehm, Dietmar/Sorace, Antonella/Bornkessel-Schlesewsky, Ina (2013): Processing flexible form-to-meaning mappings: Evidence for enriched composition as opposed to indeterminacy. In: Language and Cognitive Processes 28, S. 1244-1274.

Rösler, Frank/Röder, Brigitte/Streb, Judith (2003): Psychophysiologie semantischer und syntaktischer Verarbeitungsprozesse. In: Müller, Horst M. (Hg.): Neurokognition der Sprache. (= Neurokognition 1). Tübingen, S. 7-29.

Schmid, Monika S. et al. (2016): Designing research on bilingual development. Behavioral and neurolinguistics experiments. Cham.

Schmid, Monika S. (2016): Multi-factorial studies: Populations and linguistic features. In: Schmid, Monika S. et al., S. 13-28.

Wiedemann, H.R. (1994): Hans Berger. In: European Journal of Pediatrics 153, S. 705. 